\title{
ON THE STOCHASTIC BEVERTON-HOLT EQUATION WITH SURVIVAL RATES
}

\author{
PAUL H. BEZANDRY, TOKA DIAGANA, AND SABER ELAYDI
}

\begin{abstract}
The paper studies a Beverton-Holt difference equation, in which both the recruitment function and the survival rate vary randomly. It is then shown that there is a unique invariant density, which is asymptotically stable. Moreover, a basic theory for random mean almost periodic sequence on $\mathbb{Z}_{+}$ is given. Then, some sufficient conditions for the existence of a mean almost periodic solution to the stochastic Beverton-Holt equation are given.
\end{abstract}

\section{INTRODUCTION}

The impetus of this paper came from two sources. The first source is the paper of Haskell and Sacker [5], in which they considered a Beverton-Holt equation without a survival term, that is,

$$
x(n+1)=\frac{\mu K_{n} x(n)}{K_{n}+(\mu-1) x(n)},
$$

where $\mu>1$ and $K_{n}>0$ for each $n \in \mathbb{Z}_{+}$. The second source is a recent paper by Diagana, Elaydi, and Yakubu [2], in which a fundamental theory of almost periodic sequences on $\mathbb{Z}_{+}$has been established. Furthermore, the abstract results were then applied to study the existence of almost periodic solutions to the nonautonomous Beverton-Holt difference equation

$$
x(n+1)=\gamma_{n} x(n)+\frac{\left(1-\gamma_{n}\right) \mu K_{n} x(n)}{\left(1-\gamma_{n}\right) K_{n}+\left(\mu-1+\gamma_{n}\right) x(n)},
$$

where $\gamma_{n} \in(0,1), K_{n}>0$ are almost periodic sequences on $\mathbb{Z}_{+}$and $\mu>1$.

In this paper we study (1.2) in the case when $\left(K_{n}\right)_{n \in \mathbb{Z}_{+}}$and $\left(\gamma_{n}\right)_{n \in \mathbb{Z}_{+}}$are random sequences $[8,11]$. We then extend the theorem of Haskell-Sacker [5] to $(1.2)$ and show the existence of a unique asymptotically stable invariant density (Theorem 4.2). Based upon the results of [2], we develop a theory of random mean almost periodic sequences on $\mathbb{Z}_{+}$. The developed theory is then applied to investigate the stochastic Beverton-Holt equation (1.2). Among other things, it is shown (Theorem 3.1 ) that under some suitable assumptions, (1.2) has a unique random mean almost periodic solution on $\mathbb{Z}_{+}$.

The paper is organized as follows. In Section 2, we develop a basic theory of random mean almost periodic sequences on $\mathbb{Z}_{+}$. In Section 3 , we apply the techniques developed in Section 2 to find some sufficient conditions for the existence and uniqueness of a mean almost periodic solution to the Beverton-Holt equation

2000 Mathematics Subject Classification. Primary 39A10, 60H10; Secondary 34F05.

Key words and phrases. mean almost periodic sequence, difference equation, Beverton-Holt equation, recruitment function, survival rate, invariant density. 
(1.2). Section 4 is devoted to the study of asymptotic stability of some Markov operators associated with the Stochastic Beverton-Holt equations. Finally, in Section 5 , the paper concludes with a discussion of open problems involving attenuance and resonance for the case of two varying parameters Beverton-Holt equation with a survival rate.

\section{Preliminaries}

In this section we establish a basic theory for random mean almost periodic sequences on $\mathbb{Z}_{+}$. To facilitate our task, we first introduce the notations needed in the sequel.

Let $(\mathbb{B},\|\cdot\|)$ be a Banach space and let $(\Omega, \mathcal{F}, \mathbf{P})$ be a probability space. Throughout the rest of the paper, $\mathbb{Z}_{+}$denotes the set of all nonnegative integers. Define $L^{1}(\mathbf{P} ; \mathbb{B})$ to be the space of all $\mathbb{B}$-valued random variables $V$ such that

$$
\mathbf{E}\|V\|:=\left(\int_{\Omega}\|V\| d \mathbf{P}\right)<\infty .
$$

It is then routine to check that $L^{1}(\mathbf{P} ; \mathbb{B})$ is a Banach space when it is equipped with its natural norm $\|\cdot\|_{1}$ defined by, $\|V\|_{1}:=\mathbf{E}\|V\|$ for each $V \in L^{1}(\mathbf{P}, \mathbb{B})$.

Let $X=\left\{X_{n}\right\}_{n \in \mathbb{Z}_{+}}$be a sequence of $\mathbb{B}$-valued random variables satisfying $\mathbf{E}\left\|X_{n}\right\|<\infty$ for each $n \in \mathbb{Z}_{+}$. Thus, interchangeably we can, and do, speak of such a sequence as a function, which goes from $\mathbb{Z}_{+}$into $L^{1}(\mathbf{P} ; \mathbb{B})$.

This setting requires the following preliminary definitions.

Definition 2.1. [7] An $L^{1}(\mathbf{P} ; \mathbb{B})$-valued random sequence $X=\{X(n)\}_{n \in \mathbb{Z}_{+}}$is said to be stochastically bounded whenever

$$
\lim _{N \rightarrow \infty}\left(\sup _{n \in \mathbb{Z}_{+}} \mathbf{P}\{\|X(n)\|>N\}\right)=0 .
$$

Definition 2.2. An $L^{1}(\mathbf{P} ; \mathbb{B})$-valued random sequence $X=\{X(n)\}_{n \in \mathbb{Z}_{+}}$is said to be mean (Bohr) almost periodic if for each $\varepsilon>0$ there exists $N_{0}(\varepsilon)>0$ such that among any $N_{0}$ consecutive integers there exists at least an integer $p>0$ for which

$$
\mathbf{E}\|X(n+p)-X(n)\|<\varepsilon, \forall n \in \mathbb{Z}_{+} .
$$

An integer $p>0$ with the above-mentioned property is called an $\varepsilon$ - almost period for $X=\{X(n)\}_{n \in \mathbb{Z}_{+}}$. The collection of all $\mathbb{B}$-valued random sequences $X=\{X(n)\}_{n \in \mathbb{Z}_{+}}$which are mean (Bohr) almost periodic is then denoted by $A P\left(\mathbb{Z}_{+} ; L^{1}(\mathbf{P} ; \mathbb{B})\right)$.

Similarly, one defines the mean (Bochner) almost periodicity as follows:

Definition 2.3. An $L^{1}(\mathbf{P} ; \mathbb{B})$-valued random sequence $X=\{X(n)\}_{n \in \mathbb{Z}_{+}}$is called mean (Bochner) almost periodic if for every sequence $\{h(n)\}_{n \in \mathbb{Z}_{+}} \subset \mathbb{Z}_{+}$there exists a subsequence $\left\{h\left(k_{s}\right)\right\}_{s \in \mathbb{Z}_{+}}$such that $\left\{X\left(n+h\left(k_{s}\right)\right)\right\}_{s \in \mathbb{Z}_{+}}$converges (in the mean) uniformly with respect to $n \in \mathbb{Z}_{+}$.

Following along the same arguments as in the proof of [2, Theorem 2.4, p. 241], one can show that those two notions of almost periodicity are equivalent:

Theorem 2.4. An $L^{1}(\mathbf{P} ; \mathbb{B})$-valued random sequence $X=\{X(n)\}_{n \in \mathbb{Z}_{+}}$is mean (Bochner) almost periodic if and only if it is mean (Bohr) almost periodic. 
An important and straightforward consequence of Theorem 2.4 is the next corollary, which plays a key role in the proof of Lemma 3.2.

Corollary 2.5. If $X_{1}=\left\{X^{1}(n)\right\}_{n \in \mathbb{Z}_{+}}, X_{2}=\left\{X^{2}(n)\right\}_{n \in \mathbb{Z}_{+}}, \ldots$, and $X_{N}=$ $\left\{X^{N}(n)\right\}_{n \in \mathbb{Z}_{+}}$are $N$ random sequences, which belong to $A P\left(\mathbb{Z}_{+} ; L^{1}(\mathbf{P} ; \mathbb{B})\right)$, then for each $\varepsilon>0$ there exists $N_{0}(\varepsilon)>0$ such that among any $N_{0}(\varepsilon)$ consecutive integers there exists an integer $p>0$ for which

$$
\mathbf{E}\left\|X^{j}(n+p)-X^{j}(n)\right\|<\varepsilon
$$

for each $n \in \mathbb{Z}_{+}$and for $j=1,2, \ldots, N$.

Definition 2.6. A sequence of $\mathbb{B}$-valued random variables $X=\{X(n)\}_{n \in \mathbb{Z}_{+}}$is said to be almost periodic in probability if for each $\varepsilon>0, \eta>0$, there exists $N_{0}(\varepsilon)>0$ such that among any $N_{0}$ consecutive integers there exists at least an integer $p>0$ for which

$$
\mathbf{P}\{\omega:\|X(\omega, n+p)-X(\omega, n)\| \geq \varepsilon\}<\eta, \forall n \in \mathbb{Z}_{+} .
$$

This definition of almost periodicity in probability is similar to the concept of (Bohr) almost periodicity on $\mathbb{R}_{+}$.

Lemma 2.7. If $X$ belongs to $A P\left(\mathbb{Z}_{+} ; L^{1}(\mathbf{P} ; \mathbb{B})\right)$, then

(i) there exists a constant $M>0$ such that $\mathbf{E}\|X(n)\| \leq M$ for each $n \in \mathbb{Z}_{+}$;

(ii) $X$ is stochastically bounded; and

(iii) $X$ is almost periodic in probability.

Proof. (i) One follows along the same lines as in the proof of [2, Lemma 2.6]. Assume that $\{\mathbf{E}\|X(n)\|\}_{n \in \mathbb{Z}_{+}}$is not bounded. Then for some subsequence $\mathbf{E}\left\|X\left(n_{i}\right)\right\| \rightarrow$ $\infty$ as $i \rightarrow \infty$. Let $\varepsilon=1$. Then there exists an integer $N_{0}(\varepsilon)>0$ that satisfies the almost periodicity definition. There exists $n_{i}=s_{1}$ such that $n_{i}=s_{1}>N_{0}(\varepsilon)$ Then among the integers

$$
\left\{s_{1}-N_{0}(\varepsilon)+1, s_{1}-N_{0}(\varepsilon)+2, \ldots, s_{1}\right\}
$$

there exists $\widehat{s}_{1}$ such that

$$
\mathbf{E}\left\|X\left(n+\widehat{s}_{1}\right)-X(n)\right\|<1
$$

Next, choose $n_{j}=s_{2}$ such that $n_{j}=s_{2}>N_{0}(\varepsilon)+s_{1}$. Then among the integers

$$
\left\{s_{2}-N_{0}(\varepsilon)+1, s_{2}-N_{0}(\varepsilon)+2, . ., s_{2}\right\}
$$

there exists $\widehat{s}_{2}$ such that

$$
\mathbf{E}\left\|X\left(n+\widehat{s}_{2}\right)-X(n)\right\|<1
$$

Repeating this process, we obtain a sequence $\left\{\widehat{s}_{i}\right\} \rightarrow \infty$ as $i \rightarrow \infty$ such that

$$
\mathbf{E}\left\|X\left(n+\widehat{s}_{i}\right)-X(n)\right\|<1 \text { for } r=1,2,3, \ldots,
$$

and a subsequence $\left\{s_{i}\right\}$ of $\left\{n_{i}\right\}$ with $\left\{s_{i}\right\} \rightarrow \infty$ as $i \rightarrow \infty$. Moreover,

$$
s_{i}=\widehat{s}_{i}+u_{i}
$$

where $0 \leq u_{i}<N_{0}(\varepsilon)$.

Since $\left\{u_{i}\right\}$ is finite, there exists $u_{i_{0}}$ that is repeated infinitely many times and $s_{i_{r}}=\widehat{s}_{i_{r}}+u_{i_{0}}$, where $i_{r} \rightarrow \infty$ as $i \rightarrow \infty$. Therefore,

$$
\mathbf{E}\left\|X\left(n+\widehat{s}_{i_{r}}\right)-X\left(u_{i_{0}}\right)\right\|<1 \text {. }
$$


Moreover,

$$
\mathbf{E}\left\|X\left(n+s_{i_{r}}\right)-X\left(u_{i_{0}}\right)\right\|<1 .
$$

Hence, $\left\{X\left(s_{i_{r}}\right)\right\}$ is bounded; a contradiction.

To prove (ii), we use the Markov's inequality to obtain

$$
\sup _{n \in \mathbb{Z}_{+}} \mathbf{P}\{\|X(n)\|>N\} \leq \frac{1}{N} \sup _{n \in \mathbb{Z}_{+}} \mathbf{E}\|X(n)\| \leq \frac{M}{N},
$$

and hence

$$
\lim _{N \rightarrow \infty}\left(\sup _{n \in \mathbb{Z}_{+}} \mathbf{P}\{\|X(n)\|>N\}\right)=0
$$

Using similar arguments, we also obtain the almost periodicity in probability of X.

Let $U B\left(\mathbb{Z}_{+} ; L^{1}(\mathbf{P} ; \mathbb{B})\right)$ denote the collection of all uniformly bounded $L^{1}(\mathbf{P} ; \mathbb{B})$ valued random sequences $X=\{X(n)\}_{n \in \mathbb{Z}_{+}}$. It is then easy to check that the space $U B\left(\mathbb{Z}_{+} ; L^{1}(\mathbf{P} ; \mathbb{B})\right)$ is a Banach space when it is equipped with the norm:

$$
\|X\|_{\infty}=\sup _{n \in \mathbb{Z}_{+}} \mathbf{E}\|X(n)\|
$$

Lemma 2.8. $A P\left(\mathbb{Z}_{+} ; L^{1}(\mathbf{P} ; \mathbb{B})\right) \subset U B\left(\mathbb{Z}_{+} ; L^{1}(\mathbf{P} ; \mathbb{B})\right)$ is a closed space.

Proof. It is clear that $A P\left(\mathbb{Z}_{+} ; L^{1}(\mathbf{P} ; \mathbb{B})\right) \subset U B\left(\mathbb{Z}_{+} ; L^{1}(\mathbf{P} ; \mathbb{B})\right)$ (see (i) of Lemma 2.7). Now let $\left(X_{m}\right)_{m \in \mathbb{N}} \subset A P\left(\mathbb{Z}_{+} ; L^{1}(\mathbf{P} ; \mathbb{B})\right)$ be a random sequence such that $\left\|X_{m}-X\right\|_{\infty} \mapsto 0$ as $m \mapsto \infty$ for some $X \in U B\left(\mathbb{Z}_{+} ; L^{1}(\mathbf{P} ; \mathbb{B})\right)$. To complete the proof we have to prove that $X \in A P\left(\mathbb{Z}_{+} ; L^{1}(\mathbf{P} ; \mathbb{B})\right)$.

Since $X$ is uniformly bounded in the sense of $L^{1}(\mathbf{P} ; \mathbb{B})$, it remains to prove that it is mean almost periodic. Now, let $\varepsilon>0$ and choose $m$ such that

$$
\left\|X_{m}-X\right\|_{\infty}<\frac{\varepsilon}{3}
$$

Now since $\left(X_{m}\right)_{m \in \mathbb{N}}$ is mean almost periodic, then there exists a positive integer $N_{0}(\varepsilon)$ such that among any $N_{0}$ consecutive integers, there exists at least an integer $p>0$ for which

$$
\mathbf{E}\left\|X_{m}(n+p)-X_{m}(n)\right\|<\frac{\varepsilon}{3}, \quad \forall n \in \mathbb{Z}_{+} .
$$

Now

$$
\begin{aligned}
\mathbf{E}\|X(n+p)-X(n)\| & \leq \mathbf{E}\left\|X_{m}(n+p)-X(n+p)\right\| \\
& +\mathbf{E}\left\|X_{m}(n+p)-X_{m}(n)\right\| \\
& +\mathbf{E}\left\|X_{m}(n)-X(n)\right\| \\
& \leq \mathbf{E}\left\|X_{m}(n+p)-X_{m}(n)\right\| \\
& +2 \sup _{n \in \mathbb{Z}_{+}} \mathbf{E}\left\|X_{m}(n)-X(n)\right\| \\
& <2 \frac{\varepsilon}{3}+\frac{\varepsilon}{3} \\
& =\varepsilon
\end{aligned}
$$

and hence

$$
\sup _{n \in \mathbb{Z}_{+}} \mathbf{E}\|X(n+p)-X(n)\| \leq \varepsilon
$$


In view of the above, the space $A P\left(\mathbb{Z}_{+} ; L^{1}(\mathbf{P} ; \mathbb{B})\right)$ of random mean almost sequences equipped with the sup norm $\|\cdot\|_{\infty}$ is also a Banach space.

\section{Mean Almost Periodicity of the Solution}

In constant environments, theoretical discrete-time population models are usually formulated under the assumption that the dynamics of the total population size in generation $n$, denoted by $x(n)$, are governed by equations of the form

$$
x(n+1)=f(x(n))+\gamma x(n),
$$

where $\gamma \in(0,1)$ is the constant "probability" of surviving per generation, and the function $f: \mathbb{R}_{+} \rightarrow \mathbb{R}_{+}$models the birth or recruitment process.

Almost periodic effects can be introduced into (3.1) by writing the recruitment function or the survival probability as almost periodic sequences. This is modelled with the equation

$$
x(n+1)=f(n, x(n))+\gamma_{n} x(n),
$$

where either $\left\{\gamma_{n}\right\}_{n \in \mathbb{Z}_{+}}$or $\{f(n, x(n))\}_{n \in \mathbb{Z}_{+}}$are almost periodic and $\gamma_{n} \in(0,1)$.

In a recent paper, Franke and Yakubu [4] studied (3.2) with the periodic constant recruitment function

$$
f(n, x(n))=K_{n}\left(1-\gamma_{n}\right),
$$

and with the periodic Beverton-Holt recruitment function is

$$
f(n, x(n))=\frac{\left(1-\gamma_{n}\right) \mu K_{n} x(n)}{\left(1-\gamma_{n}\right) K_{n}+\left(\mu-1+\gamma_{n}\right) x(n)},
$$

where the carrying capacity $K_{n}$ is $p$-periodic, $K_{n+p}=K_{n}$ for all $t \in \mathbb{Z}_{+}$and $\mu>1$.

We now introduce the notations needed in the sequel. From now on we assume that both the carrying capacity $K_{n}$ and the survival rate $\gamma_{n}$ are random and that $\gamma_{n}, n \in \mathbb{Z}_{+}$are independent and independent of the sequence $\left\{K_{n}\right\}_{n \in \mathbb{Z}_{+}}$.

Let $\mathbb{B}=\mathbb{R}_{+}=[0,+\infty)$ equipped with the absolute value of $\mathbb{R}$. Our objective in this section is to find sufficient conditions for the existence of a random mean almost periodic solution of (1.2), where both $\left\{K_{n}\right\}_{n \in Z_{+}}$and $\left\{\gamma_{n}\right\}_{n \in Z_{+}}$belong to $A P\left(\mathbb{Z}_{+} ; L^{1}\left(\mathbf{P} ; \mathbb{R}_{+}\right)\right)$and $\mu>1$.

We now state our main theorem.

Theorem 3.1. Suppose that both sequences $\left\{K_{n}\right\}_{n \in Z^{+}}$and $\left\{\gamma_{n}\right\}_{n \in Z_{+}}$belong to $A P\left(\mathbb{Z}_{+} ; L^{1}\left(\mathbf{P} ; \mathbb{R}_{+}\right)\right)$and $\mu>1$. Then (1.2) has a unique random mean almost periodic solution whenever

$$
\sup _{n \in \mathbb{Z}_{+}}\left\{\mathbf{E}\left[\gamma_{n}\right]\right\}<\frac{1}{\mu+1} .
$$

The proof of Theorem 3.1 requires the following lemma.

Lemma 3.2. Let

$$
f(n, X(n))=\frac{\left(1-\gamma_{n}\right) \mu K_{n} X}{\left(1-\gamma_{n}\right) K_{n}+\left(\mu-1+\gamma_{n}\right) X(n)}
$$

where both $\left\{K_{n}\right\}_{n \in Z^{+}}$and $\left\{\gamma_{n}\right\}_{n \in Z_{+}}$belong to $A P\left(\mathbb{Z}_{+} ; L^{1}\left(\mathbf{P} ; \mathbb{R}_{+}\right)\right)$and $\mu>1$. Then,

(i) $f$ is $\mu$-Lipschitz in the following sense:

$$
\mathbf{E}|f(n, U)-f(n, V)| \leq \mu \mathbf{E}|U-V|, \quad \forall U, V \in L^{1}\left(\mathbf{P} ; \mathbb{R}_{+}\right), n \in \mathbb{Z}_{+} ;
$$


(ii) If $X$ belongs to $A P\left(\mathbb{Z}_{+} ; L^{1}\left(\mathbf{P} ; \mathbb{R}_{+}\right)\right)$, then the sequence $\{f(n, X(n))\}_{n \in \mathbb{Z}_{+}}$ also belongs to $A P\left(\mathbb{Z}_{+} ; L^{1}\left(\mathbf{P} ; \mathbb{R}_{+}\right)\right)$.

Proof. (i) It is routine to check that $|f(n, U)-f(n, V)| \leq \mu|U-V|$, and hence $\mathbf{E}|f(n, U)-f(n, V)| \leq \mu \mathbf{E}|U-V|$.

To prove (ii), set $A_{n}=\left(1-\gamma_{n}\right) K_{n}$ and $B_{n}=\mu-1+\gamma_{n}$. Then $f$ can be written as follows:

$$
f(n, X(n))=\mu \frac{A_{n} X(n)}{A_{n}+B_{n} X(n)} \text { for each } n \in \mathbb{Z}_{+} .
$$

Using the fact that $\left\{\gamma_{n}\right\},\left\{K_{n}\right\}$, and $\{X(n)\}$ are mean almost periodic and making use of respectively Lemma 2.7(i) and Corollary 2.5, we can choose a constant $M>0$ such that $\mathbf{E}\left|K_{n}\right|<M$ for all $n \in \mathbb{Z}_{+}$and for each $\varepsilon>0$ there exists a positive integer $N_{0}(\varepsilon)$ such that among any $N_{0}(\varepsilon)$ consecutive integers, there exists an integer $p>0$, a common $\varepsilon$-almost period for $\left\{\gamma_{n}\right\},\left\{K_{n}\right\}$, and $\{X(n)\}$, for which

$$
\mathbf{E}\left|\gamma_{n+p}-\gamma_{n}\right| \leq \frac{\varepsilon(\mu-1)^{2}}{3 \mu^{2} M}, \quad \mathbf{E}\left|K_{n+p}-K_{n}\right| \leq \frac{\varepsilon(\mu-1)}{6 \mu^{2}}
$$

and

$$
\mathbf{E}|X(n+p)-X(n)| \leq \frac{\varepsilon}{6 \mu}
$$

for all $n \in \mathbb{Z}_{+}$.

We now evaluate $|f(n+p, X(n+p))-f(n, X(n))|$. We have:

$$
\begin{aligned}
& |f(n+p, X(n+p))-f(n, X(n))| \\
& \leq \mu\left|\frac{A_{n+p} X(n+p)}{A_{n+p}+B_{n+p} X(n+p)}-\frac{A_{n+p} X(n)}{A_{n+p}+B_{n+p} X(n+p)}\right| \\
& \quad+\mu\left|\frac{A_{n+p} X(n)}{A_{n+p}+B_{n+p} X(n+p)}-\frac{A_{n+p} X(n)}{A_{n+p}+B_{n+p} X(n)}\right| \\
& \quad+\mu\left|\frac{A_{n+p} X(n)}{A_{n+p}+B_{n+p} X(n)}-\frac{A_{n} X(n)}{A_{n}+B_{n} X(n)}\right| \\
& \leq \mu|X(n+p)-X(n)|+A_{n+p} X(n) \\
& \quad \times\left|\frac{1}{A_{n+p}+B_{n+p} X(n+p)}-\frac{1}{A_{n+p}+B_{n+p} X(n)}\right| \\
& \quad+\mu\left|\frac{A_{n+p} X(n)}{A_{n+p}+B_{n+p} X(n)}-\frac{A_{n} X(n)}{A_{n}+B_{n} X(n)}\right| \\
& \leq \mu|X(n+p)-X(n)| \\
& \quad+\mu \frac{A_{n+p}}{A_{n+p}+B_{n+p} X(n+p)} \cdot \frac{B_{n+p} X(n)}{A_{n+p}+B_{n+p} X(n)}|X(n+p)-X(n)| \\
& \quad+\mu\left|\frac{A_{n+p} X(n)}{A_{n+p}+B_{n+p} X(n)}-\frac{A_{n} X(n)}{A_{n}+B_{n} X(n)}\right| \cdot
\end{aligned}
$$

But 


$$
\begin{aligned}
\left|\frac{A_{n+p} X(n)}{A_{n+p}+B_{n+p} X(n)}-\frac{A_{n} X(n)}{A_{n}+B_{n} X(n)}\right| & \leq \mu\left|\frac{\left(A_{n+p} B_{n}-A_{n} B_{n+p}\right) X(n)^{2}}{B_{n+p} B_{n} X(n)^{2}}\right| \\
& =\mu\left|\frac{A_{n+p}}{B_{n+p}}-\frac{A_{n}}{B_{n}}\right| .
\end{aligned}
$$

Thus,

$$
|f(n+p, X(n+p))-f(n, X(n))| \leq 2 \mu|X(n+p)-X(n)|+\mu\left|\frac{A_{n+p}}{B_{n+p}}-\frac{A_{n}}{B_{n}}\right|,
$$

which in turn implies that

$\mathbf{E}|f(n+p, X(n+p))-f(n, X(n))| \leq 2 \mu \mathbf{E}|X(n+p)-X(n)|+\mu \mathbf{E}\left|\frac{A_{n+p}}{B_{n+p}}-\frac{A_{n}}{B_{n}}\right|$. We now evaluate carefully $\mathbf{E}\left|\frac{A_{n+p}}{B_{n+p}}-\frac{A_{n}}{B_{n}}\right|$ using the hypothesis of independence of the random sequence $\left\{\gamma_{n}\right\}_{n \in \mathbb{Z}_{+}}$. We have:

$$
\begin{aligned}
& \mathbf{E}\left|\frac{A_{n+p}}{B_{n+p}}-\frac{A_{n}}{B_{n}}\right|=\mathbf{E}\left|\frac{\left(1-\gamma_{n+p}\right) K_{n+p}}{\mu-1+\gamma_{n+p}}-\frac{\left(1-\gamma_{n}\right) K_{n}}{\mu-1+\gamma_{n}}\right| \\
& =\mathbf{E}\left[\frac{1}{\left(\mu-1+\gamma_{n+p}\right)\left(\mu-1+\gamma_{n}\right)} \mid(\mu-1)\left[K_{n+p}-K_{n}\right]-\gamma_{n} \gamma_{n+p}\left[K_{n+p}-K_{n}\right]\right. \\
& \left.-(\mu-1)\left[\gamma_{n+p} K_{n+p}-\gamma_{n} K_{n}\right]+\left[\gamma_{n} K_{n+p}-\gamma_{n+p} K_{n}\right] \mid\right] \\
& =\mathbf{E}\left[\frac{1}{\left(\mu-1+\gamma_{n+p}\right)\left(\mu-1+\gamma_{n}\right)} \mid(\mu-1)\left[K_{n+p}-K_{n}\right]-\gamma_{n} \gamma_{n+p}\left[K_{n+p}-K_{n}\right]\right. \\
& \left.-(\mu-1) K_{n+p}\left[\gamma_{n+p}-\gamma_{n}\right]+\gamma_{n}\left[K_{n+p}-K_{n}\right]+\gamma_{n}\left[K_{n+p}-K_{n}\right]-\left[\gamma_{n+p}-\gamma_{n}\right] \mid\right] \\
& =\mathbf{E}\left[\mid \frac{\mu-1}{\left(\mu-1+\gamma_{n+p}\right)\left(\mu-1+\gamma_{n}\right)}\left[K_{n+p}-K_{n}\right]\right. \\
& -\frac{\gamma_{n} \gamma_{n+p}}{\left(\mu-1+\gamma_{n+p}\right)\left(\mu-1+\gamma_{n}\right)}\left[K_{n+p}-K_{n}\right] \\
& -\frac{\mu-1}{\left(\mu-1+\gamma_{n+p}\right)\left(\mu-1+\gamma_{n}\right)} K_{n+p}\left[\gamma_{n+p}-\gamma_{n}\right] \\
& +\frac{(\mu-1) \gamma_{n}}{\left(\mu-1+\gamma_{n+p}\right)\left(\mu-1+\gamma_{n}\right)}\left[K_{n+p}-K_{n}\right] \\
& -\frac{\gamma_{n}}{\left(\mu-1+\gamma_{n+p}\right)\left(\mu-1+\gamma_{n}\right)}\left[K_{n+p}-K_{n}\right] \\
& -\frac{1}{\left(\mu-1+\gamma_{n+p}\right)\left(\mu-1+\gamma_{n}\right)} K_{n}\left[\gamma_{n+p}-\gamma_{n}\right] \\
& \left.\leq \frac{1}{\mu-1} \mathbf{E}\left|K_{n+p}-K_{n}\right|\right]+\mathbf{E}\left|K_{n+p}-K_{n}\right|+\frac{1}{\mu-1} \mathbf{E}\left|K_{n+p}\right| \mathbf{E}\left|\gamma_{n+p}-\gamma_{n}\right| \\
& +\mathbf{E}\left|K_{n+p}-K_{n}\right|+\frac{1}{\mu-1} \mathbf{E}\left|K_{n+p}-K_{n}\right|+\frac{1}{(\mu-1)^{2}} \mathbf{E}\left|K_{n}\right| \mathbf{E}\left|\gamma_{n+p}-\gamma_{n}\right| . \\
& \left.\leq \frac{2 \mu}{\mu-1} \mathbf{E}\left|K_{n+p}-K_{n}\right|\right]+\frac{\mu}{(\mu-1)^{2}} M \cdot \mathbf{E}\left|\gamma_{n+p}-\gamma_{n}\right| .
\end{aligned}
$$


By combining, we obtain:

$$
\begin{aligned}
\mathbf{E}|f(n+p, X(n+p))-f(n, X(n))| & \leq 2 \mu \mathbf{E}|X(n+p)-X(n)| \\
& +\frac{2 \mu^{2}}{\mu-1} \mathbf{E}\left|K_{n+p}-K_{n}\right| \\
& +\left(\frac{\mu}{(\mu-1)}\right)^{2} M \cdot \mathbf{E}\left|\gamma_{n+p}-\gamma_{n}\right| \\
& \leq \frac{\varepsilon}{3}+\frac{\varepsilon}{3}+\frac{\varepsilon}{3}=\varepsilon .
\end{aligned}
$$

We now prove Theorem 3.1.

Proof. By Lemma 3.2(ii), if $u \in A P\left(\mathbb{Z}_{+}, L^{1}\left(\mathbf{P} ; \mathbb{R}_{+}\right)\right.$, then $n \rightarrow f(n, u(n))$ belongs to $A P\left(\mathbb{Z}_{+}, L^{1}\left(\mathbf{P} ; \mathbb{R}_{+}\right)\right)$. Define the nonlinear operator $\Gamma$ by setting:

$$
\Gamma: A P\left(\mathbb{Z}_{+}, L^{1}\left(\mathbf{P} ; \mathbb{R}_{+}\right)\right) \mapsto A P\left(\mathbb{Z}_{+}, L^{1}\left(\mathbf{P} ; \mathbb{R}_{+}\right)\right),
$$

where

$$
\Gamma u(n):=\sum_{r=0}^{n-1}\left(\prod_{s=r}^{n-1} \gamma_{s}\right) f(r, u(r))
$$

It is clear that $\Gamma$ is well defined. Now, let $u, v \in A P\left(\mathbb{Z}_{+}, L^{1}\left(\mathbf{P} ; \mathbb{R}_{+}\right)\right)$having the same property as $x$ defined in the Beverton-Holt equation. Since $\left\{\gamma_{n}, n \in \mathbb{Z}_{+}\right\}$are independent and independent of $u$ and $v$, one can easily see that

$$
\mathbf{E}|\Gamma u(n)-\Gamma v(n)| \leq \sum_{r=0}^{n-1}\left\{\left(\prod_{s=r}^{n-1} \mathbf{E}\left|\gamma_{s}\right|\right) \mathbf{E}|f(r, u(r))-f(r, v(r))|\right\},
$$

and hence letting $\beta=\sup _{n \in \mathbb{Z}_{+}} \mathbf{E}\left[\gamma_{n}\right]$ we obtain

$$
\sup _{n \in \mathbb{Z}_{+}} \mathbf{E}|\Gamma u(n)-\Gamma v(n)| \leq\left(\frac{\mu \beta}{1-\beta}\right) \sup _{n \in \mathbb{Z}_{+}} \mathbf{E}|u(n)-v(n)| \text {. }
$$

Obviously, $\Gamma$ is a contraction whenever $\frac{\mu \beta}{1-\beta}<1$. In that event, using the Banach fixed point theorem it easily follows that $\Gamma$ has a unique fixed point, $\bar{x}$, which obviously is the unique mean almost periodic solution of (1.2).

\section{Asymptotic Stability of Markov Operators}

This section examines the asymptotic behavior of the stochastic Beverton-Holt equation (1.2).

Let $L^{1}(E, \nu)$ be the space of integrable functions on a measure space $(E, \mathcal{E}, \nu)$ equipped with its natural norm given by $\|f\|_{1}=\int_{E} f(x) d \nu$ and let

$$
\mathcal{D}(E):=\left\{f \in L^{1}(E, \nu): f \geq 0 \text { and } \int f d \nu=1\right\}
$$

be the space of all densities on $E$. 
Definition 4.1. Let $\mathcal{Q}$ be a Markov operator $\mathcal{Q}: L^{1}(E, \nu) \rightarrow L^{1}(E, \nu)$. Then $\left\{\mathcal{Q}^{n}\right\}$ is said to be asymptotically stable if there exists $f^{*} \in \mathcal{D}$ for which $\mathcal{Q} f^{*}=f^{*}$ and for all $f \in \mathcal{D}$

$$
\lim _{n \rightarrow \infty}\left\|\mathcal{Q}^{n} f-f^{*}\right\|_{1}=0 .
$$

As in Section 3, we suppose that both carrying the capacity $K_{n}$ and the survival rate $\gamma_{n}$ are random and for all $n,\left(K_{n}, \gamma_{n}\right)$ is chosen independently of $\left(x(0), K_{0}, \gamma_{0}\right)$, $\left(x(1), K_{1}, \gamma_{1}\right), \ldots .,\left(x(n-1), K_{n-1}, \gamma_{n-1}\right)$ from a distribution with density $\psi(k, \gamma)$ However, the survival rates $\gamma_{n}, n \in \mathbb{Z}_{+}$are no longer assumed to be independent in this section. The joint density of $x(n), K_{n}$, and $\gamma_{n}$ is then $f_{n}(x) \psi(k, \gamma)$, where $f_{n}$ is the density of $x(n)$. Furthermore, we suppose that

$$
\mathbf{E}\left|K_{n}\right|<\infty, \quad \mathbf{E}|x(0)|<\infty,
$$

and $k^{2} \psi(k, \gamma)$ is bounded above independently of $\gamma$ and that $\psi$ is supported on $\left[k_{\min }, \infty\right) \times\left[\gamma_{\min }, \infty\right)$ for some $k_{\min }>0$ and $\gamma_{\min }>0$. Moreover, we suppose that there is some interval $\left(k_{l}, k_{u}\right) \subset \mathbb{R}_{+}$on which $\psi$ is positive everywhere for all $\gamma$.

Let $h$ be an arbitrary bounded and measurable function on $\mathbb{R}_{+}$and define $b\left(K_{n}, \gamma_{n}, x(n)\right)$ to be equal to the right-hand side of equation (1.2). The expected value of $h$ at time $\mathrm{n}+1$ is then given by

$$
\mathbf{E}[h(x(n+1))]=\int_{0}^{\infty} h(x) f_{n+1}(x) d x .
$$

Furthermore, because of (1.2) and the fact that the joint density of $x(n), K_{n}$, and $\gamma_{n}$ is just $f_{n}(x) \psi(k, \gamma)$, we also have

$$
\begin{aligned}
\mathbf{E}[h(x(n+1))] & =\mathbf{E}\left[h\left(b\left(K_{n}, \gamma_{n}, x(n)\right)\right]\right. \\
& =\int_{0}^{\infty} \int_{0}^{1} \int_{0}^{\infty} h\left(b(k, \gamma, y) f_{n}(y) \psi(k, \gamma) d y d \gamma d y\right.
\end{aligned}
$$

Let us define $k=k(x, \gamma, y)$ by the equation

$$
x=\frac{(1-\gamma) \mu k y}{(1-\gamma) k+(\mu-1+\gamma) y}+\gamma y .
$$

Solving explicitly this equation for $k$ yields

$$
k=\frac{(\mu-1+\gamma) y(x-\gamma y)}{(1-\gamma)[\mu y-(x-\gamma y)]}
$$

By a change of variables, this can be written as

$$
\mathbf{E}[h(x(n+1))]=\iiint_{\{(x, \gamma, y): 0<x-\gamma y<\mu y\}} h(x) f_{n}(y) \psi(k, \gamma) \frac{1}{\frac{d b(k, \gamma, y)}{d k}} d x d \gamma d y .
$$

A simple calculation yields

$\mathbf{E}[h(x(n+1))]=\mu \int_{0}^{\infty} h(x)\left\{\iint_{A} \frac{1-\gamma}{\mu-1+\gamma} \frac{1}{(x-\gamma y)^{2}} f_{n}(y) k^{2} \psi(k, \gamma) d \gamma d y\right\} d x$,

where

$$
A=\{(\gamma, y): 0<x-\gamma y<\mu y\} .
$$

Equating the above equations, and using the fact that $h$ was an arbitrary, bounded, measurable function, we immediately obtain 


$$
f_{n+1}(x)=\mu \iint_{A} \frac{1-\gamma}{\mu-1+\gamma} \frac{1}{(x-\gamma y)^{2}} f_{n}(y) k^{2} \psi(k, \gamma) d \gamma d y .
$$

Let $\mathcal{P}: L^{1}\left(\mathbb{R}_{+}\right) \rightarrow L^{1}\left(\mathbb{R}_{+}\right)$be defined by

$$
\mathcal{P} f(x)=\mu \iint_{A} \frac{1-\gamma}{\mu-1+\gamma} \frac{1}{(x-\gamma y)^{2}} f(y) k^{2} \psi(k, \gamma) d \gamma d y,
$$

where $k=k(x, \gamma, y)$ is defined by (4.4) and $A$ in (4.5).

We can now state the main theorem of this section.

Theorem 4.2. The Markov operator $\mathcal{P}: L^{1}\left(\mathbb{R}_{+}\right) \rightarrow L^{1}\left(\mathbb{R}_{+}\right)$defined by (4.6) is asymptotically stable. $\mathcal{P}$.

Before embarking on the proof of Theorem 4.2 we first give some properties of

Lemma 4.3. The following holds:

(i) $\mathcal{P}$ is nonnegative;

(ii) $\mathcal{P}$ is a Markov operator;

(iii) If $f$ is integrable on $\mathbb{R}_{+}$and supported on $\left[k_{\min }, \infty\right)$, then so is $\mathcal{P} f$.

Proof. Clearly, $\mathcal{P}$ is nonnegative Also, $\|\mathcal{P} f\|_{1}=\|f\|_{1}$. To see this, we need to compute $\|\mathcal{P} f\|_{1}$. By interchanging the integral signs, we can write:

$$
\|\mathcal{P} f\|_{1}=\mu \int_{0}^{\infty} f(y)\left\{\int_{0}^{1} \int_{\gamma y}^{(\gamma+\mu) y} \frac{1-\gamma}{\mu-1+\gamma} \frac{1}{(x-\gamma y)^{2}} k^{2} \psi(k, \gamma) d x d \gamma\right\} d y .
$$

By letting $z=k(x, \gamma, y)$ and using the fact that $\psi$ is a density, we can show easily that

$$
\|\mathcal{P} f\|_{1}=\int_{0}^{\infty}\left\{\int_{0}^{1} \int_{0}^{\infty} \psi(z, \gamma) d z d \gamma\right\} d y=\int_{0}^{\infty} f(y) d y=\|f\|_{1} .
$$

Therefore, $\mathcal{P}$ is a Markov operator.

We now define the stochastic kernel corresponding to $\mathcal{P}$. Let $L: \mathbb{R}_{+} \times \mathbb{R}_{+} \rightarrow \mathbb{R}$ be defined by

$$
L(x, y)=\int_{0}^{1} \frac{1-\gamma}{\mu-1+\gamma} \frac{1}{(x-\gamma y)^{2}} k^{2} \psi(k, \gamma) \chi_{A}(y) d \gamma,
$$

where $A$ is defined in (4.5).

To prove (iii), note first that for any $y>x$,

$$
k(x, \gamma, y)=\frac{(\mu-1+\gamma)(x-\gamma y)}{(1-\gamma)\left(\gamma+\mu-\frac{x}{y}\right)}<x .
$$

If $f$ is integrable and supported on $\left[k_{\min }, \infty\right)$, then we have:

$$
\mathcal{P} f(x)=\int_{k_{\min }}^{\infty} L(x, y) f(y) d y .
$$

Now, suppose $x<k_{\min }$. Then for any $y \geq k_{\min }$, we have $k(x, \gamma, y)<x<k_{\min }$. Thus, $\psi(k, \gamma)=0$ for any $0<\gamma<1$. It follows that $L(x, y)=0$ and that $\mathcal{P} f(x)=0$. 
Let $L_{m}$ denote the kernel of $\mathcal{P}^{m}$. To obtain an expression for $L_{m}$ we define $b^{m}:\left(\mathbb{R}_{+} \times(0,1)\right)^{m} \rightarrow \mathbb{R}$ as follows

$$
\begin{aligned}
b^{1}\left(k_{0}, \gamma_{0}, x\right) & =b\left(k_{0}, \gamma_{0}, x\right) \\
b^{2}\left(k_{1}, \gamma_{1}, k_{0}, \gamma_{0}, x\right) & =b\left(k_{1}, \gamma_{1}, b\left(k_{0}, \gamma_{0}, x\right)\right) \\
& \vdots \\
b^{m}\left(k_{m-1}, \gamma_{m-1}, \cdots, k_{0}, \gamma_{0}, x\right) & =b\left(k_{m-1}, \gamma_{m-1}, b^{m-1}\left(k_{m-2}, \gamma_{m-2}, \cdots, k_{0}, \gamma_{0}, x\right)\right) .
\end{aligned}
$$

To lighten the notation, set $b_{m-2, \cdots, 0}^{m-1}(k, \gamma, y)=b^{m-1}\left(k_{m-2}, \gamma_{m-2}, \cdots, k_{0}, \gamma_{0}, y\right)$. Now if we let $x=b\left(k, \gamma, b_{m-2, \cdots, 0}^{m-1}(k, \gamma, y)\right)$, then, by induction, it can be shown that

$$
\gamma \gamma_{0} \cdots \gamma_{m-2} y<x<(\mu+\gamma)\left(\mu+\gamma_{0}\right) \cdots\left(\mu+\gamma_{m-2}\right) y
$$

As before, we can derive the expression for $L_{m}$ for $x$ and $y$ satisfying the inequality (4.9) and obtain:

$$
\begin{aligned}
L_{m}(x, y)= & \int_{0}^{1}\left\{\frac { ( 1 - \gamma ) \mu } { \mu - 1 + \gamma } \int \int \cdots \int \int \left[\frac{1}{\left(x-\gamma b_{m-2, \cdots, 0}^{m-1}(k, \gamma, y)\right)^{2}} \tilde{k}^{2} \times\right.\right. \\
& \left.\left.\psi(\tilde{k}, \gamma) \psi\left(k_{m-2}, \gamma_{m-2}\right) \cdots \psi\left(k_{0}, \gamma_{0}\right)\right] d k_{m-2} d \gamma_{m-2} \cdots d k_{0} d \gamma_{0}\right\} d \gamma
\end{aligned}
$$

where the integration inside the brackets is taken over the set

$\left\{\left(k_{0}, \gamma_{0}, \cdots, k_{m-2}, \gamma_{m-2}\right): 0<x-\gamma b_{m-2, \cdots, 0}^{m-1}(k, \gamma, y)<\mu b_{m-2, \cdots, 0}^{m-1}(k, \gamma, y)\right\}$

and the function $\tilde{k}$ is given by

$$
x=b^{m}\left(k, \gamma, k_{m-2}, \gamma_{m-2}, \cdots, k_{0}, \gamma_{0}, y\right) .
$$

The following lemma will play a key role in this section.

Lemma 4.4. $\mathcal{P}: L^{1}\left[k_{\min }, \infty\right) \rightarrow L^{1}\left[k_{\min }, \infty\right)$ is asymptotically stable.

Proof. To establish the asymptotic stability of $\mathcal{P}$, it suffices (see Lasota-Mackey [8]) to show that there exist an integer $m$, a function $g \in L^{1}\left[k_{\min }, \infty\right)$, and an interval $(\alpha, \beta)$ such that for all $x, y \in L^{1}\left[k_{m i n}, \infty\right)$,

$$
L_{m}(x, y) \leq g(x)
$$

and for all $x \in(\alpha, \beta)$ and $y \in\left[k_{\min }, \infty\right)$,

$$
L_{m}(x, y)>0 \text {. }
$$

We first verify the first condition. By the Mean Value Theorem, there exists a number $\widehat{\gamma}, \gamma_{\min }<\widehat{\gamma}<1$ such that

$$
\begin{aligned}
L_{m}(x, y)= & \left(1-\gamma_{\min }\right) \frac{(1-\widehat{\gamma}) \mu}{\mu-1+\widehat{\gamma}} \iint \cdots \iint\left[\frac{1}{\left(x-\widehat{\gamma} b_{m-2, \cdots, 0}^{m-1}(k, \gamma, y)\right)^{2}} \tilde{k}^{2} \times\right. \\
& \left.\psi(\tilde{k}, \widehat{\gamma}) \psi\left(k_{m-2}, \gamma_{m-2}\right) \cdots \psi\left(k_{0}, \gamma_{0}\right)\right] d k_{m-2} d \gamma_{m-2} \cdots d k_{0} d \gamma_{0},
\end{aligned}
$$

where the integration inside the brackets is taken over the set $\left\{\left(k_{0}, \gamma_{0}, \cdots, k_{m-2}, \gamma_{m-2}\right): 0<x-\gamma b_{m-2, \cdots, 0}^{m-1}(k, \gamma, y)<\mu b_{m-2, \cdots, 0}^{m-1}(k, \gamma, y)\right\}$.

Now, choose a number $\delta$ such that

$$
0<\delta<\frac{(\mu-1)(1-\widehat{\gamma})}{\mu-1+\widehat{\gamma}} k_{\min }
$$


and write $L_{m}(x, y)$ as follows

$$
L_{m}(x, y)=L_{m}^{1}(x, y)+L_{m}^{2}(x, y)
$$

where

$$
\begin{aligned}
L_{m}^{1}(x, y)= & \left(1-\gamma_{\min }\right) \frac{(1-\widehat{\gamma}) \mu}{\mu-1+\widehat{\gamma}} \iint \cdots \iint\left[\frac{1}{\left(x-\widehat{\gamma} b_{m-2, \cdots, 0}^{m-1}(k, \gamma, y)\right)^{2}} \tilde{k}^{2} \times\right. \\
& \left.\psi(\tilde{k}, \widehat{\gamma}) \psi\left(k_{m-2}, \gamma_{m-2}\right) \cdots \psi\left(k_{0}, \gamma_{0}\right)\right] d k_{m-2} d \gamma_{m-2} \cdots d k_{0} d \gamma_{0},
\end{aligned}
$$

where the integration inside the brackets is taken over the set

$\left\{\left(k_{0}, \gamma_{0}, \cdots, k_{m-2}, \gamma_{m-2}\right): \delta<x-\widehat{\gamma} b_{m-2, \cdots, 0}^{m-1}(k, \gamma, y)<\mu b_{m-2, \cdots, 0}^{m-1}(k, \gamma, y)\right\}$

and

$$
\begin{aligned}
L_{m}^{2}(x, y)= & \left(1-\gamma_{\min }\right) \frac{(1-\widehat{\gamma}) \mu}{\mu-1+\widehat{\gamma}} \iint \cdots \iint\left[\frac{1}{\left(x-\widehat{\gamma} b_{m-2, \cdots, 0}^{m-1}(k, \gamma, y)\right)^{2}} \tilde{k}^{2} \times\right. \\
& \left.\psi(\tilde{k}, \widehat{\gamma}) \psi\left(k_{m-2}, \gamma_{m-2}\right) \cdots \psi\left(k_{0}, \gamma_{0}\right)\right] d k_{m-2} d \gamma_{m-2} \cdots d k_{0} d \gamma_{0},
\end{aligned}
$$

where the integration inside the brackets is taken over the set

$$
\left\{\left(k_{0}, \gamma_{0}, \cdots, k_{m-2}, \gamma_{m-2}\right): 0<x-\widehat{\gamma} b_{m-2, \cdots, 0}^{m-1}(k, \gamma, y)<\delta\right\} .
$$

We need to evaluate $L_{m}^{1}(x, y)$ and $L_{m}^{2}(x, y)$ separately. Let us start with $L_{m}^{2}(x, y)$. Using the explicit expression for $\tilde{k}$, that is

$$
k=\frac{(\mu-1+\widehat{\gamma}) b_{m-2, \cdots, 0}^{m-1}(k, \gamma, y)\left(x-\widehat{\gamma} b_{m-2, \cdots, 0}^{m-1}(k, \gamma, y)\right)}{(1-\widehat{\gamma})\left[\mu b_{m-2, \cdots, 0}^{m-1}(k, \gamma, y)-\left(x-\widehat{\gamma} b_{m-2, \cdots, 0}^{m-1}(k, \gamma, y)\right)\right]}
$$

We can easily show that if the inequality $0<x-\tilde{\gamma} b_{m-2, \ldots, 0}^{m-1}(k, \gamma, y)<\delta$ holds, then

$$
k<\frac{(\mu-1+\widehat{\gamma}) b_{m-2, \cdots, 0}^{m-1}(k, \gamma, y) \delta}{(1-\widehat{\gamma})\left(\mu b_{m-2, \cdots, 0}^{m-1}(k, \gamma, y)-\delta\right)} .
$$

Since $\delta<k_{\text {min }} \leq b_{m-2, \ldots, 0}^{m-1}(k, \gamma, y)$ (because $\left.y \geq k_{m i n}\right)$, we can write

$$
k<\frac{(\mu-1+\widehat{\gamma}) \delta}{(1-\widehat{\gamma})(\mu-1)} .
$$

The definition of $\delta$ allows us to bound the right-hand side of this inequality to obtain $k<k_{\text {min }}$, so that $\psi(k, \gamma)=0$ and $L_{m}^{2}(x, y)=0$.

As to $L_{m}^{1}(x, y)$, we introduce a function $g:\left[k_{\min }, \infty\right) \rightarrow \mathbb{R}_{+}$defined as follows

$$
g(x)=\frac{\mu}{\mu-1}\left\{\sup _{k \in \mathbb{R}_{+}} \psi(k, \widehat{\gamma})\right\}\left\{\sup _{k_{m-2}, \gamma_{m-2}, \ldots, k_{0}, \gamma_{0}, y} \frac{1}{\left(x-\widehat{\gamma} b_{m-2, \cdots, 0}^{m-1}(k, \gamma, y)\right)^{2}}\right\} .
$$

Clearly, by the Monotone Convergence Theorem, $g \in L^{1}\left[k_{\min }, \infty\right)$. Also, for all $x, y \in\left[k_{\min }, \infty\right), L_{m}^{1}(x, y) \leq C g(x)$. By combining these two evaluations, we obtain:

for all $m$.

$$
L_{m}(x, y) \leq C g(x)
$$

As to the second condition, note that, for all $k \in \mathbb{R}_{+}, \gamma \in(0,1)$, and $x \in$ $\left[k_{\min }, \infty\right), b^{n}(k, \gamma, \cdots, k, \gamma, x) \rightarrow k$ as $n \rightarrow \infty$. Now, since

$$
\lim _{n \rightarrow \infty} b^{n}\left(k_{l}, \gamma, \cdots, k_{l}, \gamma, y\right)=k_{l}
$$


and

$$
\lim _{n \rightarrow \infty} b^{n}\left(k_{u}, \gamma, \cdots, k_{u}, \gamma, y\right)=k_{u}
$$

one can choose $m \in \mathbb{N}$ large enough such that

$$
b^{m}\left(k_{l}, \gamma, \cdots, k_{l}, \gamma, y\right)<\frac{k_{l}+k_{u}}{2}
$$

and

$$
b^{m}\left(k_{u}, \gamma, \cdots, k_{u}, \gamma, y\right)>\frac{k_{l}+k_{u}}{2}
$$

for all $y \geq k_{\min }$ and $\gamma \in\left(\gamma_{\min }, 1\right)$.

Set $\alpha=\frac{k_{l}+k_{u}}{2}$ and $\beta=b^{m}\left(k_{u}, \gamma, \cdots, k_{u}, \gamma, k_{\min }\right)$. If $x \in(\alpha, \beta)$ and $y \in$ $\left[k_{\min }, \infty\right)$, the inequalities (4.11) and (4.12) imply that

$$
b^{m}\left(k_{l}, \gamma, \cdots, k_{l}, \gamma, y\right)<x<b^{m}\left(k_{u}, \gamma, \cdots, k_{u}, \gamma, y\right) .
$$

Since $b^{m}$ is continuous in all variables it follows by the implicit function theorem that there exists an open ball $B \subset\left(k_{l}, k_{u}\right)^{m-1}$ and a function $\kappa: B \rightarrow\left(k_{l}, k_{u}\right)$ such that for all $\left(k_{0}, k_{1}, \cdots, k_{m-2}\right) \in B, k=\kappa\left(k_{0}, k_{1}, \cdots, k_{m-2}\right)$ is a solution of $x=b^{m}\left(k, \gamma, k_{m-2}, \gamma_{m-2}, \cdots, k_{0}, \gamma_{0}, y\right)$. It follows that $L_{m}(x, y)>0$. This completes the proof of the lemma.

The next step is to investigate how $\mathcal{P}$ acts on the parts of the density whose support is in $\left[0, k_{\min }\right]$.

The following lemma deals with this situation.

Lemma 4.5. If $f \in L^{1}\left(\mathbb{R}_{+}\right)$, then

$$
\lim _{m \rightarrow \infty} \int_{0}^{k_{\min }} \mathcal{P}^{m} f(x) d x=0 .
$$

Although the proof of this lemma follows along the same line as the one in Haskell-Sacker, its complete details are given in this paper for convenience.

Proof. Choose a number $c$ such that $0<c<k_{\min }$ and $b\left(k_{l}, \gamma, c\right)>k_{\min }$ for all $\gamma$

Also, define a number $y_{c}^{m}$ for which $b^{m}\left(k_{\min }, \gamma_{\min }, \ldots, k_{\min }, \gamma_{\min }, y_{c}^{m}\right)=c$. Note that $y_{c}^{m} \rightarrow 0$ as $m$ goes to infinity.

We can then write:

$$
\begin{aligned}
\int_{0}^{k_{\min }} \mathcal{P}^{m} f(x) d x & =\int_{0}^{c} \int_{y_{c}^{m}}^{\infty} f(y) L_{m}(x, y) d x d y+\int_{0}^{c} \int_{0}^{y_{c}^{m}} f(y) L_{m}(x, y) d x d y \\
& +\int_{c}^{k_{\min }} \mathcal{P}^{m} f(x) d x .
\end{aligned}
$$

Let us evaluate the first term. Note that if $y>y_{c}^{m}$ and $k_{0}, k_{1}, \ldots, k_{m-1} \geq k_{\min }$, then $b^{m}\left(k_{m-1}, \gamma_{\min }, \ldots, k_{0}, \gamma_{\min }, y\right) \geq b^{m}\left(k_{\min }, \gamma_{\min }, \ldots, k_{\min }, \gamma_{\min }, y_{c}^{m}\right)=c$. Thus, if $y>y_{c}^{m}$ and $x=b^{m}\left(k_{m-1}, \gamma_{m-1}, \ldots, k_{0}, \gamma_{0}, y\right)<c$, then

$$
b^{m}\left(k_{m-1}, \gamma_{\min }, \ldots, k_{0}, \gamma_{\min }, y\right)<c
$$

which, in turn, implies that at least one of the $k_{i}$ 's must be less than $k_{\min }$. Therefore, for any such $x$ and $y$, the integrand in the definition of $L_{m}(x, y)$ is equal to zero. 
The first term is then zero. As far as the second term is concerned, we can bound it to obtain:

$$
\int_{0}^{c} \int_{0}^{y_{c}^{m}} L_{m}(x, y) f(y) d y d x \leq \int_{0}^{y_{c}^{m}} f(y) \int_{0}^{\infty} L_{m}(x, y) d x d y=\int_{0}^{y_{c}^{m}} f(y) d y \rightarrow 0
$$

as $m \rightarrow \infty$.

As to the last term, we need to prove that

$$
\lim _{m \rightarrow \infty} \int_{c}^{k_{\min }} P^{m} f(x) d x=0 .
$$

Since $\mathcal{P}$ is a Markov operator, we can write:

$$
\int_{c}^{\infty} \mathcal{P}\left(f \chi_{\left[c, k_{\min }\right]}\right)(x) d x=\left\|\mathcal{P}\left(f \chi_{\left[c, k_{\min }\right)}\right)\right\|=\left\|f \chi_{\left[c, k_{\min }\right)}\right\|=\int_{c}^{k_{\min }} f(x) d x .
$$

Let now $q=\int_{0}^{1} \int_{k_{l}}^{k_{u}} \psi(k, \gamma) d k d \gamma$ and for any $y$, set

$$
B_{y}=\left\{x: \exists k \in\left(k_{l}, k_{u}\right): x \geq b(k, \gamma, y) \text { for all } \gamma\right\} .
$$

If $y \geq c$ and $x \in B_{y}$, then there exists a $k \in\left(k_{l}, k_{u}\right)$ such that $x \geq b(k, \gamma, y)$ for all $\gamma$, which implies that $x \geq b\left(k_{l}, \gamma, c\right)>k_{\min }$. Thus, $B_{y} \subset\left[k_{\min }, \infty\right)$. Therefore, for any $y \in\left[c, k_{\min }\right)$, we have:

$$
\begin{aligned}
\int_{k_{\min }}^{\infty} L(x, y) d x & \geq \int_{0}^{1} \int_{B_{y}} \frac{1-\gamma}{\mu-1+\gamma} \frac{1}{(x-\gamma y)^{2}} k^{2} \psi(k, \gamma) d x d \gamma \\
& \geq \int_{0}^{1} \int_{k_{l}}^{k_{u}} \psi(k, \gamma) d k d \gamma=q
\end{aligned}
$$

The last inequality was obtained by using the appropriate substitution.

By combining, we have

$$
\begin{aligned}
\int_{c}^{k_{\min }} \mathcal{P} f(x) d x & =\int_{c}^{\infty} \mathcal{P}\left(f \chi_{\left[c, k_{\min }\right)}\right)(x) d x-\int_{k_{\min }}^{\infty} \mathcal{P}\left(f \chi_{\left[c, k_{\min }\right)}\right) d x \\
& \leq \int_{c}^{k_{\min }} f(x) d x-q \int_{c}^{k_{\min }} f(x) d x \\
& =(1-q) \int_{c}^{k_{\min }} f(x) d x
\end{aligned}
$$

It follows by induction that

$$
\int_{c}^{k_{\min }} \mathcal{P}^{m} f(x) d x \leq(1-q)^{m} \int_{c}^{k_{\min }} f(x) d x \rightarrow 0
$$

as $m \rightarrow \infty$.

This will complete the proof in the case when $f$ is nonnegative. For a general $f \in L^{1}\left(\mathbb{R}_{+}\right)$, this result can be obtained by considering its positive and negative parts.

The proof of Theorem 4.2 follows along the same line as the one in HaskellSacker [5] using Lemmas 4.3, 4.4, and 4.5. It is omitted. 


\section{REMARKS}

Haskell and Sacker [5] showed that for every initial non-zero state variable and almost all random sequences of carrying capacities $K_{n}$, the average of the state variable along an orbit $x(n)$ is strictly less than the average of the carrying capacities $K_{n}$ (attenuance in mean). If one assumes that $\gamma_{n}=\gamma$ is constant in (1.2), then it may be shown that the same result holds, that is, (1.2) is attenuant in the mean. However, if both parameters $\gamma_{n}$ and $K_{n}$ are random sequences, we are still unable to determine either attenuance in the mean or resonance in the mean, that is for almost every $\omega \in \Omega$ and every $x \in \mathbb{R}_{+}$,

$$
\begin{aligned}
\lim _{n \rightarrow \infty} \frac{1}{n} \sum_{i=0}^{n-1} K_{i}(\omega) & >\lim _{n \rightarrow \infty} \frac{1}{n} \sum_{i=0}^{n-1} x_{i}(\omega, x) \text { (Attenuance) } \\
& <\lim _{n \rightarrow \infty} \frac{1}{n} \sum_{i=0}^{n-1} x_{i}(\omega, x) \text { (Resonance) }
\end{aligned}
$$

The problem of attenuance or resonance has not been yet resolved even when $\gamma_{n}$ and $K_{n}$ are periodic sequences on $\mathbb{Z}_{+}$, (see for example Elaydi-Sacker [3], and FrankeYakubu [4]). It would be of great interest to develop criteria for attenuance and resonance for the case of two varying parameters Beverton-Holt equation with a survival rate.

\section{REFERENCES}

1. C. Corduneanu, Almost Periodic Functions, 2nd Edition, Chelsea-New York, 1989.

2. T. Diagana, S. Elaydi and A-A. Yakubu, Population Models in Almost Periodic Environments. J. Difference Equations and Appl. 13 (2007), no. 4, 239-260.

3. S. Elaydi and R. Sacker, Nonautonomous Beverton-Holt Equations and the Cushing-Henson Conjectures. J. Difference Equations and Appl. 11 (2005), 337-347.

4. J. Franke and A-A Yakubu, Population Models with Periodic Recruitment Functions and Survival Rates. J. Difference Equations and Appl. 11 (2005), no. 14, 1169-1184

5. C. Haskell and R. Sacker, The Stochastic Beverton-Holt Equation and the M. Neubert Conjecture. J. Dynam. Diffential Eq. 17 (2005), no. 4, 825-844

6. T. Kawata, Almost periodic weakly stationary processes. Statistics and probability: essays in honor of C. R Rao, pp. 383-396, North-Holland, Amsterdam-New York, 1982.

7. J. Luo, Stochastically bounded solutions of a nonlinear stochastic differential equations. $J$. Comput. Appl. Math. 196 (2006), no. 1, 87-93.

8. A. Lasota and M.C. Mackey, Chaos, Fractals and Noise-Stochastic Aspects of Dynamics. Applied Mathematics Sciences, v.97. Springer, New York, 2nd Edition, 1994

9. E. Slutsky, Sur les fonctions aléatoires presque périodiques et sur la decomposition des functions aléatoires, Actualités Sceintifiques et industrielles, 738, 33-55, Herman. Paris, 1938.

10. R. J. Swift, Almost Periodic Harmonizable Processes, Georgian Math J. 3 (1996), no. 3, 275-292.

11. M. Udagawa, Asymptotic properties of distributions of some functionals of random variables. Rep. Statist Appl. Res. Union Jap. Sci. Eng. 2 (1952). no. 2-3, 1-98

Department of Mathematics, Howard University, Washington, DC 20059

E-mail address: pbezandry@howard.edu

Department of Mathematics, Howard University, Washington, DC 20059

E-mail address: tdiagana@howard.edu

Department of Mathematics, Trinity University, San Antonio, TX 78212

E-mail address: selaydi@trinity.edu 